\title{
Viscosity Measurements of Dilute Poly(2-ethyl-2-oxazoline) Aqueous Solutions Near Theta Temperature Analyzed within the Joint Rouse-Zimm Model
}

\author{
Jana Tóthová, ${ }^{1}$ Katarína Paulovičová, ${ }^{2}$ and Vladimír Lisý, ${ }^{1,3}$ \\ ${ }^{1}$ Department of Physics, Technical University of Košice, Park Komenského 2, 04200 Košice, Slovakia \\ ${ }^{2}$ Institute of Experimental Physics, Slovak Academy of Sciences, Watsonova 47, 04001 Košice, Slovakia \\ ${ }^{3}$ Laboratory of Radiation Biology, Joint Institute of Nuclear Research, Dubna, Moscow Oblast 141 980, Russia
}

Correspondence should be addressed to Jana Tóthová; jana.tothova@tuke.sk and Vladimír Lisý; vladimir.lisy@tuke.sk

Received 5 January 2015; Accepted 20 February 2015

Academic Editor: Cornelia Vasile

Copyright (C) 2015 Jana Tóthová et al. This is an open access article distributed under the Creative Commons Attribution License, which permits unrestricted use, distribution, and reproduction in any medium, provided the original work is properly cited.

\begin{abstract}
The steady-state shear viscosity of low-concentrated Poly(2-ethyl-2-oxazoline) (PEOX) aqueous solutions is measured near the presumed theta temperature using the falling ball viscometry technique. The experimental data are analyzed within the model that joins the Rouse and Zimm bead-spring theories of the polymer dynamics at the theta condition, which means that the polymer coils are considered to be partially permeable to the solvent. The polymer characteristics thus depend on the draining parameter $h$ that is related to the strength of the hydrodynamic interaction between the polymer segments. The Huggins coefficient was found to be 0.418 at the temperature $20^{\circ} \mathrm{C}$, as predicted by the theory. This value corresponds to $h=2.92$, contrary to the usual assumption of the infinite $h$. This result indicates that the theta temperature for the PEOX water solutions is $20^{\circ} \mathrm{C}$ rather than $25^{\circ} \mathrm{C}$ in the previous studies. The experimental intrinsic viscosity is well described coming from the Arrhenius equation for the shear viscosity.
\end{abstract}

\section{Introduction}

For the study of the behavior and conformations of polymer chains in solution, various viscosimetry techniques are widely used. By measuring the viscosity of the solutions, they in principle allow determining important polymer parameters, such as its relaxation times or the gyration and hydrodynamic radii $[1,2]$. However, the interpretation of such experiments encounters serious difficulties. This is due to problems with the treatment of the experimental data $[3,4]$ but also because of fundamental problems with the theory of viscosity of polymer solutions $[5,6]$. A particular problem concerns the so called theta temperature. In a dilute polymer solution at the Flory theta temperature $T_{\theta}$ the repulsive effects of the volume interactions are cancelled by the attractive forces between the polymer segments, and the polymer coils behave like ideal chains forming random coils. The polymer parameters are then easily calculated and compared to experimentally measured quantities. Due to this much work being devoted to the determination of $T_{\theta}$, for the methods in this way see, for example, $[1,2,7]$. However, different methods could lead to different $T_{\theta}[7]$. Moreover, often reliable values of $T_{\theta}$ are not known, since they are outside the range of the temperatures at which the experiments on polymer solutions are conducted or even the temperatures when the polymer chains exist [811]. In such cases the interpretation of the experiments and the determination of the polymer parameters become difficult and ambiguous. To make a comparison between the theory and experiment, $T_{\theta}$ should be well defined.

In the present work we investigate Poly(2-ethyl-2oxazoline) (also known as PEOX), the polymer, for which water is assumed to be nearly a theta solvent at room temperature $\left(\approx 25^{\circ} \mathrm{C}\right)$ [12]. The intrinsic viscosity scales with the molecular mass $M$ approximately as $\sim M^{0.56}$, with the exponent close to 0.6 , as predicted for theta conditions by the Mark-Houwink equation for very high $M$ polymers adopt ideally a Gaussian form conformation [13] (see also the results presented in [14] and references there).

The chemical and physical properties of PEOX were described long ago [12]. It is a nonionic tertiary amide 
synthetic water- and organic-soluble polymer, which belongs to the group of Poly(oxazolines). PEOX is biocompatible and heat stable and blends well with other polymers [14]. Due to these properties it finds many applications [15], including the use in thermosensitive materials, as sensors, in drug delivery systems [16-20] and others. Viscosity measurements indicate that the PEOX chains at room temperature behave almost ideally [12]. PEOX also exhibits lower critical solution temperature behavior in water $[21,22]$. This behavior was determined by the decrease in second virial coefficient with increasing temperature and is attributed to hydrogen bonding between the PEOX molecules and the water molecules. The excess enthalpy and excess entropy of mixing in water are both negative and have magnitudes which increase with molecular weight [21]. These properties have been attributed to partial organization of the PEOX-water system through specific hydrogen bonding interactions between the carbonyl oxygen on the PEOX side chain and water molecules in solution. In [14], one can find the information concerning the interactions between the PEOX chains and solvents, which affect the polymer conformational behavior. The fact that PEOX in water forms a typical random coil conformation, which is energetically favorable, was proven by extensive investigations of its behavior in solution, including also the static light scattering and molecular modeling techniques.

Despite a number of papers devoted to the physicochemical studies of PEOX, to our knowledge, the detailed comparison between the experiments and existing theories of the dynamics of dilute polymer solutions is absent.

It is the aim of the present work to make a careful interpretation of the viscometric experiments on the PEOX solutions and to determine the main phenomenological parameters of the polymer. We come from the Zimm beadspring model [23], which was generalized by us to include the hydrodynamic interactions (HI) between the beads without the assumption of the impermeability of the polymer coil with respect to the solvent. Contrary to the usual approach [2] to the interpretation of experimental data, we do not consider the polymer dynamics in its nondraining limit, which is the opposite case to the free-draining or fully permeable Rouse model [24]. Only for infinitely strong HI the coil behaves as an impenetrable body. In general, the theory should contain the Rouse and Zimm models as limiting cases of small (Rouse) and large (Zimm) HI $[25,26]$. The strength of the HI is related to the draining parameter $h$, which appeared already in the famous work by Kirkwood and Riseman [27]. Our approach thus goes back to this classical work but corrects its result for the intrinsic viscosity. To describe the experiments, usually the draining effects are ignored. It will be demonstrated that the "nonuniversal" theory presented in Section 3 much better describes the obtained experimental data.

\section{Experimental}

The Poly(2-ethyl-2-oxazoline) (PEOX) in our experiments was a commercial product of Sigma-Aldrich Co. (Germany) without other modifications. The weight-average molecular weight was 500,000. The polymer was dissolved in deionized water. The apparatus used was an automated microviscometer (AMVn), combined with a density meter (DMA $4500 \mathrm{M}$ ), both from Anton Paar, Austria. The measurement principle of AMVn is the falling ball system consisting of the detection of a ball's drop time in a diagonally mounted glass capillary filled with sample. The shear rate is influenced by changing the inclination angle of the capillary. The measurement system configuration with the capillary and ball diameters $1.6 \mathrm{~mm}$ and $1.5 \mathrm{~mm}$, respectively, was used. It is suitable for the measuring range from 0.3 to about $10 \mathrm{mPa} \cdot \mathrm{s}$ with reproducibility better than $0.5 \%$. The temperature is measured with an uncertainty $0.05^{\circ} \mathrm{C}$. The experimental data were treated with the software OriginPro (OriginLab, USA).

Since we were interested in the behavior of the PEOX solution near the theta temperature, the measurements were carried out at 20,25 , and $30^{\circ} \mathrm{C}$. The polymer concentration $c$ changed from 0.5 to $4 \mathrm{mg} / \mathrm{mL}$, that is, well below the concentration $1 /[\eta]$ (for the intrinsic viscosity $[\eta]$ determined in the works $[12,14]$ and also in our experiments). The sample thus can be considered as a dilute solution and up to $c$ about $2 \mathrm{mg} / \mathrm{mL}$ (which was estimated in [14] as the critical concentration $c^{* *}$ [28-30] corresponding with the first permanent contacts between macromolecules in solution) even as an extremely dilute one. In the treatment of the experimental data the effect of overlapping of different coils thus plays a minor role.

The experimental results are shown in Figures 1 and 2 . Let us denote the viscosity of the solution as $\eta$ and that of the solvent as $\eta_{0}$. The relative viscosity, $\eta_{\text {rel }}=\eta / \eta_{0}$, showed no dependence on the shear rate, so that the solution can be treated as a Newtonian fluid. The results for $\eta_{\text {rel }}$ for different temperatures and concentrations are presented in Figure 1. The curves are second-order polynomial fits $\eta_{\text {rel }}=1+B_{1} c+$ $B_{2} c^{2}$. The standard errors of the coefficients are shown in the parenthesis: $1\left(<3 \times 10^{-3}\right), B_{1}\left(<3 \times 10^{-3} \mathrm{~mL} / \mathrm{mg}\right)$, and $B_{2}$ $\left(<7 \times 10^{-4} \mathrm{~mL}^{2} / \mathrm{mg}^{2}\right)$. The fits match the curves obtained by simple linking of the experimental points.

At low concentrations the Huggins equation is used to describe the dependence on the polymer concentration $c$ $[1,2]$ :

$$
\eta_{\mathrm{red}}=\frac{\eta_{\mathrm{sp}}}{c}=\frac{\left(\eta_{\mathrm{rel}}-1\right)}{c}=[\eta]\left(1+k_{H}[\eta] c+\cdots\right) .
$$

Here, $\eta_{\text {red }}$ and $\eta_{\mathrm{sp}}$ are the reduced and specific viscosity, respectively, $[\eta]$ is the intrinsic viscosity, and $k_{H}$ is the Huggins coefficient. Alternatively, the Kraemer expression for the viscosity is used,

$$
\ln \eta_{\text {rel }}=c[\eta]\left(1+k_{K}[\eta] c+\cdots\right),
$$

where $k_{K}$ is Kraemer's constant. At low $c$ approximately the equation $k_{K} \approx 1 / 2-k_{H}$ should be obeyed. Figure 2 shows the dependences of the viscosity functions $\eta_{\mathrm{sp}} / c$ and $c^{-1} \ln \eta_{\text {rel }}$ on the concentration for three experimental temperatures.

The experimental dependences on $c$ are well fitted by linear polynomials $A+B c$, with the errors in the determination of the intercept $A$ being less than $3.5 \times 10^{-3}$ times smaller than $A$. The slopes of the lines, $B$, are determined with the errors 


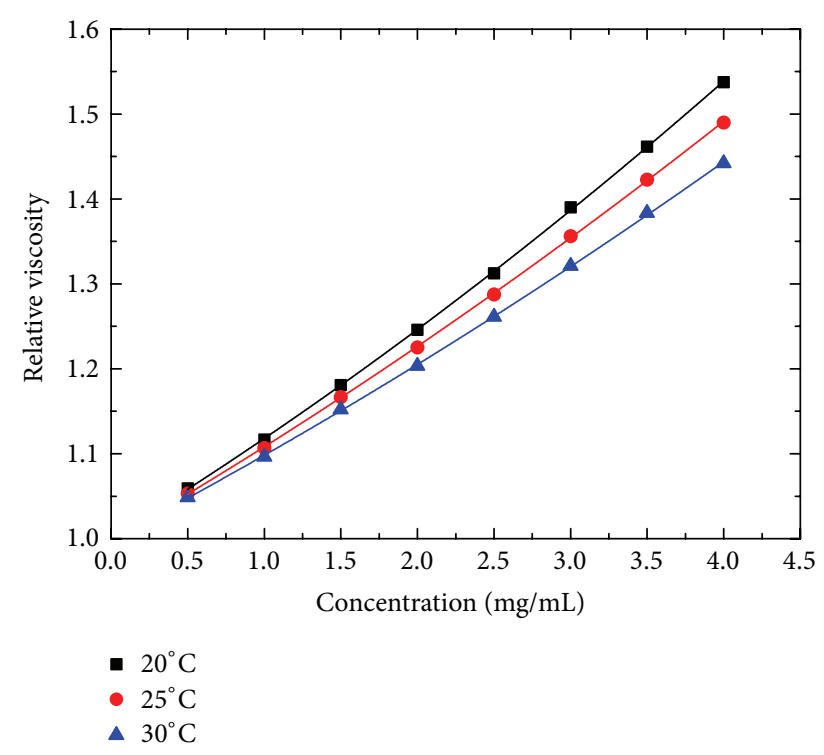

FIGURE 1: Relative viscosity of the PEOX 500,000 aqueous solutions at different concentrations and temperatures.

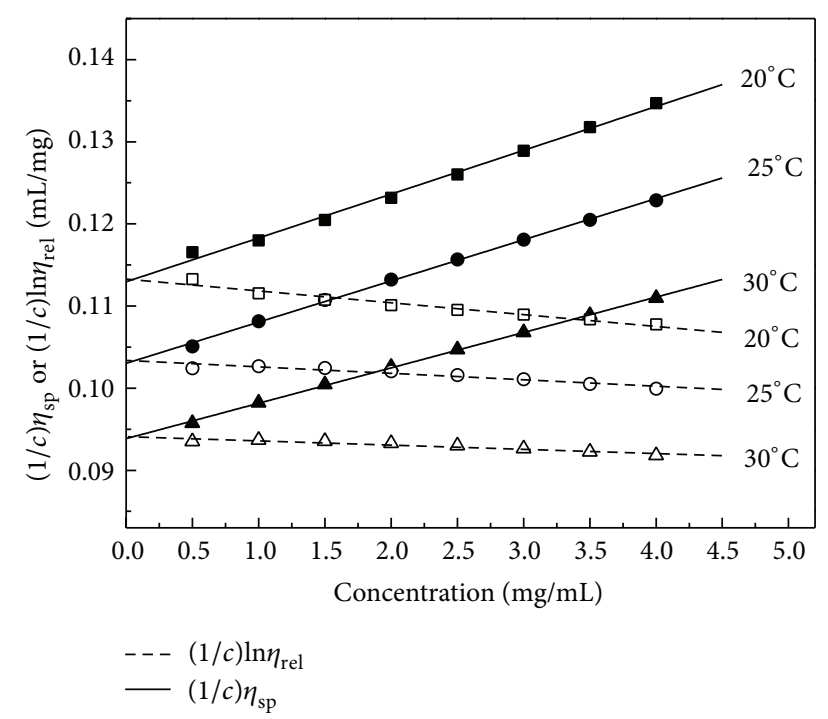

Figure 2: Huggins and Kraemer viscosity functions from dilute to extremely dilute PEOX solutions.

$3 \times 10^{-2}$ and $8 \times 10^{-2}$ times smaller than $B$ in the Huggins and Kraemer functions, respectively. The quantities $A$ and $B$ were used to calculate the intrinsic viscosities $[\eta]_{H}$ and $[\eta]_{K}$ and the constants $k_{H}$ and $k_{K}$ for different temperatures, as shown in Table 1 . In parenthesis, the values are given that were obtained without fitting the relative viscosity by the second-order polynomial. For every experimental value of the viscosity the Huggins and Kraemer functions were calculated and only then the linear fits were used to get the intrinsic viscosities and the constants $[\eta]_{H}$ and $[\eta]_{K}$. It is seen that the two approaches give close results lying within the experimental errors.
TABLE 1: Intrinsic viscosities and Huggins and Kraemer constants of PEOX solutions.

\begin{tabular}{lccccc}
\hline$T\left({ }^{\circ} \mathrm{C}\right)$ & $k_{H}$ & $k_{K}$ & $k_{H}+k_{K}$ & $\begin{array}{c}{[\eta]_{H}} \\
\mathrm{~mL} / \mathrm{mg}\end{array}$ & $\begin{array}{c}{[\eta]_{K}} \\
\mathrm{~mL} / \mathrm{mg}\end{array}$ \\
\hline \multirow{2}{*}{20} & 0.418 & 0.112 & 0.530 & 0.113 & 0.113 \\
& $(0.418)$ & $(0.113)$ & $(0.531)$ & $(0.113)$ & $(0.113)$ \\
25 & 0.472 & 0.073 & 0.545 & 0.103 & 0.103 \\
& $(0.453)$ & $(0.087)$ & $(0.540)$ & $(0.103)$ & $(0.104)$ \\
30 & 0.489 & 0.060 & 0.549 & 0.094 & 0.094 \\
& $(0.464)$ & $(0.078)$ & $(0.542)$ & $(0.094)$ & $(0.095)$ \\
\hline
\end{tabular}

\section{Theory}

In the interpretation of the viscometry experiments on dilute polymer solutions usually the "universal" Flory theory of steady-state processes [1], which is consistent with the theory of Zimm [23] and that of Kirkwood and Riseman [27] in its nondraining limit, has been widely used for a long time. However, various experiments indicate a nonuniversality of these theories, possibly caused by draining effects usually ignored by experimentalists. The first clear observation of the draining effect for long polymer chains, using static and dynamic light scattering experiments and viscometry, was reported in [31]. For more experiments revealing nonuniversality effects in the polymer dynamics, see $[32,33]$. In the calculation of the viscosity of polymer liquids these effects can be accounted for based on the Brinkman-DebyeBueche theory $[26,34,35]$ of the long-time relaxation of the internal modes of flexible polymers. In this theory the polymer solution is described as a porous medium permeable to the solvent flow. This flow is governed by the linearized Navier-Stokes equation with an additional term $-\kappa^{2} \eta_{0} \vec{v}$ (the average value of the force acting on the liquid in an element of volume $\mathrm{d} V$, provided that the average number of polymers in solution per $\mathrm{d} V$ is $c$ ), where $1 / \kappa^{2}, \eta_{0}$, and $\vec{v}$ are the solvent permeability, viscosity, and velocity, respectively. It holds that $\kappa^{2} \eta_{0}=c(t) f$, where $f$ is the friction factor on one polymer chain that can be determined from the Einstein relation for the diffusion coefficient of the polymer coil, $D=k_{B} T / f$. The polymer is assumed to consist of $N \gg 1$ beads connected by elastic forces that are given by the Gaussian equilibrium distribution of the beads [2]. The motion of the solvent created by the motion of beads is much faster than the motion of the coils, which determines the changes of $c(t)$. Thus, these changes around the equilibrium value $c$, which are due to the motion of the coils, are neglected. We do not a priori assume the validity of a concrete, Rouse or Zimm, model of the polymer dynamics. Only the strength of the hydrodynamic interactions (HI) determines which type of the polymer behavior is dominant. With the increase of $c$ the Zimm contribution to the observable quantities (such as the coil diffusion coefficient or the viscosity of the solution) decreases and the polymer tends to behave (as distinct from the previous theories [2]) in correspondence with the Rouse model. For infinitely diluted solutions at theta conditions such theory was developed in $[26,36,37]$. Now the diffusion and the relaxation of the polymer internal modes depend on $c$. 
The diffusion coefficient is a sum of the Zimm and Rouse contributions (independent on $c$ ), and $D(c)=D_{Z}(c)+D_{R}$, where $D_{R}$ and $D_{Z}(0)$ are the well-known Rouse and Zimm limits for the diffusion coefficients [2]. For what follows we need the relaxation times of the internal modes. In the stationary case they relax exponentially, with the rates

$$
\frac{1}{\tau_{p}(c)}=\frac{1}{\tau_{p R}}+\frac{1}{\tau_{p Z}(c)}, \quad p=1,2,3, \ldots,
$$

where $\tau_{p R}$ and $\tau_{p Z}(0) \equiv \tau_{p Z}$ are given by [2]

$$
\begin{aligned}
\tau_{p R} & =\frac{2 N^{2} a^{2} b \eta_{0}}{\pi k_{B} T p^{2}}, \\
\tau_{p Z} & =\frac{(\sqrt{N} a)^{3} \eta_{0}}{\left(3 \pi p^{3}\right)^{1 / 2} k_{B} T} .
\end{aligned}
$$

Here, $b$ is the radius of the bead and $a$ is the mean square distance between the beads along the chain and, at $c \rightarrow 0$,

$$
\tau_{p Z}(c)=\tau_{p Z}\left(1+\frac{N}{6 \pi p} \kappa^{2} a^{2}-\cdots\right) .
$$

In the opposite case, when $c \rightarrow \infty$,

$$
\tau_{p Z}(c) \approx \frac{1}{2} \tau_{p Z} \chi_{p}=\frac{\left(N a^{2}\right)^{2} \eta_{0}}{6 \pi k_{B} T p^{2}} \kappa .
$$

The quantity $h(p)=\tau_{p R} / \tau_{p Z}=h p^{-1 / 2}$, with the draining parameter $h=2(3 N / \pi)^{1 / 2} b / a$, indicates the strength of the $\mathrm{HI}$, that is, whether the dynamics are close to the pure Zimm $(h \gg 1)$ or Rouse $(h \ll 1)$ type. Using the relaxation times $\tau_{p}$, the steady state viscosity of the solution can be calculated from the formula $[5,38]$

$$
\eta(c)=\eta_{0}+\frac{1}{2} k_{B} T c \sum_{p=1}^{\infty} \tau_{p}(c) .
$$

In the Rouse limit we obtain the known result [2] $\eta(c)=\eta_{0}+$ $\pi N^{2} a^{2} b c \eta_{0} / 6$. In the Zimm case at low concentrations [26]

$$
\begin{aligned}
\frac{\eta(c)-\eta_{0}}{\eta_{0}}= & \frac{c}{2 \sqrt{3 \pi}}\left(N a^{2}\right)^{3 / 2} \\
& \cdot \sum_{p=1}^{\infty} p^{-3 / 2}\left[1+\frac{\sqrt{6 \pi}}{16 p} c\left(N a^{2}\right)^{3 / 2}+\cdots\right] \\
= & 0.425 c\left(N a^{2}\right)^{3 / 2}\left[1+0.140 c\left(N a^{2}\right)^{3 / 2}+\cdots\right],
\end{aligned}
$$

where the first term corresponds to the known formula [2]. A general formula for the viscosity for arbitrary $h$ was derived in [26]. For very low concentrations of the chains it gives for the intrinsic viscosity

$$
[\eta]=\lim _{c \rightarrow 0} \frac{\eta(c)-\eta_{0}}{\eta_{0} c}=\frac{1}{\pi} N^{2} a^{2} b \sum_{p=1}^{\infty} \frac{1}{p^{2}}\left(1+\frac{h}{\sqrt{p}}\right)^{-1}
$$

and for the Huggins coefficient in (1)

$$
\begin{aligned}
k_{H}= & \pi h\left(1+\frac{4 \sqrt{2} h}{3}\right)^{-1}\left[\sum_{p=1}^{\infty} \frac{1}{p^{2}}\left(1+\frac{h}{\sqrt{p}}\right)^{-1}\right]^{-2} \\
& \cdot \sum_{p=1}^{\infty} \frac{1}{p^{7 / 2}}\left(1+\frac{h}{\sqrt{p}}\right)^{-2} .
\end{aligned}
$$

From (9) at large $h$ (the Zimm case) one finds

$$
[\eta]=\frac{N^{3 / 2} a^{3}}{2 \sqrt{3 \pi}} \sum_{p=1}^{\infty} p^{-3 / 2}=3 \sqrt{\frac{2}{\pi}} R_{G}^{3} \zeta\left(\frac{3}{2}\right) \approx 6.253 R_{G}^{3}
$$

where $\zeta$ is the Riemann zeta function [39]. In this case $k_{H}=$ $3 \pi 2^{-5 / 2} \zeta(5 / 2) \zeta^{-2}(3 / 2) \approx 0.3275$. (Note that in [26] the factor $1 / 2$ is missing in this expression.) This result differs from the known results (e.g., Doi and Edwards [2] give the value 0.757, $k_{H}=0.6949$ in [40], etc.). In the opposite Rouse limit when $h \rightarrow 0, k_{H}$ approaches zero as $k_{H} \approx \pi h \zeta(3.5) \zeta^{-2}(2) \approx 1.3 h$. For convenience, in this section $c$ was the number of polymers per unit volume and $[\eta]$ has the dimension of volume. In the more common volume/mass units $[\eta]$ for Zimm polymers will have the form

$$
[\eta]=\frac{N^{3 / 2} a^{3}}{(12 \pi)^{1 / 2}} \frac{N_{A}}{M} \sum_{p=1}^{\infty} p^{-3 / 2}=\Phi_{0} \frac{N^{3 / 2} a^{3}}{M},
$$

where $N_{A}$ is Avogadro's number, $M$ is the polymer molar mass, and $\Phi_{0}=2.56 \times 10^{23}$ is the Flory-Fox factor. This value results from several approximations [2]. More exact numerical calculations give slightly different values $\Phi_{0}=$ $2.66 \times 10^{23}[6]$. For arbitrary strength of HI, $[\eta]$ should be a function of $h$. The dependence of $[\eta]$ on $h$ for theta solvents can be written as in (12), but, using (9), with $\Phi_{0}$ replaced by the function

$$
\Phi(h)=\Phi_{0}\left(\sum_{p=1}^{\infty} p^{-3 / 2}\right)^{-1} \sum_{p=1}^{\infty} \frac{p^{-3 / 2}}{1+p^{1 / 2} h^{-1}} .
$$

This result is similar to that of Kirkwood and Riseman [27] but with a difference due to a different $h$ (their value of $h$ is $\sqrt{ } 2$ times smaller).

Thus, having measured the viscosity of a polymer solution, the above formulas (9)-(12) can be used to determine the intrinsic viscosity and then the polymer size. In theta solutions the polymer relaxation times, as well as the combination $N^{1 / 2} a$ or the gyration radius $R_{G}=(N / 6)^{1 / 2} a$, can be found assuming the Flory-Fox factor is known. Alternatively, if $R_{G}$ is known, the function $\Phi(h)$ can be determined from $[\eta]$, and from (13) the draining parameter $h$ can be extracted.

\section{Comparison of the Theory and Experiment}

In our experiments, $[\eta]$ near the assumed theta point at $25^{\circ} \mathrm{C}$ changes around $0.1 \mathrm{~mL} / \mathrm{mg}$; see Table 1 . This is in good agreement with the result obtained in [12] and the Mark-Houwink equation deduced there, $[\eta]=0.65 \times 10^{-4} M^{0.56} \mathrm{~mL} / \mathrm{mg}$, if 
$M=500,000$. In [14], the coefficient 0.57 was found instead of 0.65 . However, there is no evidence that at $T=25^{\circ} \mathrm{C}$ the solvent is exactly at the $\theta$ condition. Since at $T_{\theta}$ the exponent in the Mark-Houwink equation should be 0.6 giving a larger $[\eta]$, based on the data of Table 1 , we rather expect that the theta temperature should be lower than $25^{\circ} \mathrm{C}$. This is supported also by our theory. Let us compare the theoretical value for the Huggins coefficient with the one determined from the experimental data. In the region of the expected theta temperature, $k_{H}$ changes from 0.418 to 0.489 . According to the theory, its value 0.3275 in the nondraining (Zimm) limit is notably lower. This indicates that the studied polymer coils are not perfectly impermeable to the solvent and behave partially as Rouse polymers. The Huggins coefficient $k_{H}$ as a function of the draining parameter $h$ is given by (10). Using Mathematica [41], we found that between the limiting Rouse $\left(k_{H}=0\right)$ and Zimm value $k_{H}(h)$ represents a peak with the maximum $k_{H}=0.417$ at $h \approx 2.92$; see Figure 3 . This maximal $k_{H}$ is very close to the experimentally determined value 0.418 at $T=20^{\circ} \mathrm{C}$. It thus suggests that the real theta temperature of the aqueous PEOX solution is $20^{\circ} \mathrm{C}$ rather than $25^{\circ} \mathrm{C}$.

Using the found $h$, we calculate the Flory-Fox function (13) $\Phi(2.92)=0.58454 \Phi_{0}$. Equation (12) for the experimental value $[\eta]=0.113 \mathrm{~m}^{3} / \mathrm{kg}$ and $M=500 \mathrm{~kg} / \mathrm{mol}$ gives an estimation for the PEOX gyration radius of the coils $R_{G}=$ $(N / 6)^{1 / 2} a=29.1 \mathrm{~nm}$.

If the polymer coil is regarded as a sphere of volume $V$, from the familiar Einstein formula for the viscosity of suspensions, $\eta-\eta_{0}=(5 / 2) \eta_{0} \phi$ [42] ( $\phi$ is the volume fraction of polymer chains in solution), the intrinsic viscosity and the hydrodynamic (viscometric) radius are expressed as

$$
\begin{aligned}
{[\eta] } & =2.5 \frac{V N_{A}}{M}, \\
R_{H} & =\left(\frac{3[\eta] M}{10 \pi N_{A}}\right)^{1 / 3} .
\end{aligned}
$$

The equation gives $R_{H}=20.8 \mathrm{~nm}$, close to the Zimm value $19.4 \mathrm{~nm}$ at $h \rightarrow \infty$. Both these values lie in the interval of $R_{H}$ from $18.0 \mathrm{~nm}$ to $24.7 \mathrm{~nm}$, obtained from the dependence of the hydrodynamic radius on molecular weight, as it was empirically determined for PEOX polymers in [43], with the use of a combination of analytical ultracentrifugation and laser light scattering (note that the scaling law found in [43] again indicates that PEOX polymers adopt a random coil conformation in aqueous solution at room temperatures). Within the joint Rouse-Zimm theory for a finite $h$ it holds $\pi^{1 / 2} R_{G} / R_{H}=8 / 3+2^{1 / 2} / h$, so that one obtains a lower value $R_{H}=16.4 \mathrm{~nm}$.

Finally, let us assume that the shear viscosity of the studied solution is a thermally activated process. Using the Arrhenius equation to describe the temperature dependence of the viscosity (see [44] and references therein), we have $\eta=A \exp (B / T)$. Here, $T$ is the absolute temperature, $B=$ $B_{0}+\alpha(M) \mathcal{c}$, and $\ln \left(A / A_{0}\right)=\beta(M) \mathcal{c}$, with $A_{0}$ and $B_{0}$ being the values for the solvent. By comparison of this formula with the representation of viscosity at $c \rightarrow 0$ in (1), $\eta=$ $\eta_{0}(1+[\eta] c+\cdots)$, one finds $[\eta] \approx \beta+\alpha / T$. The experimental

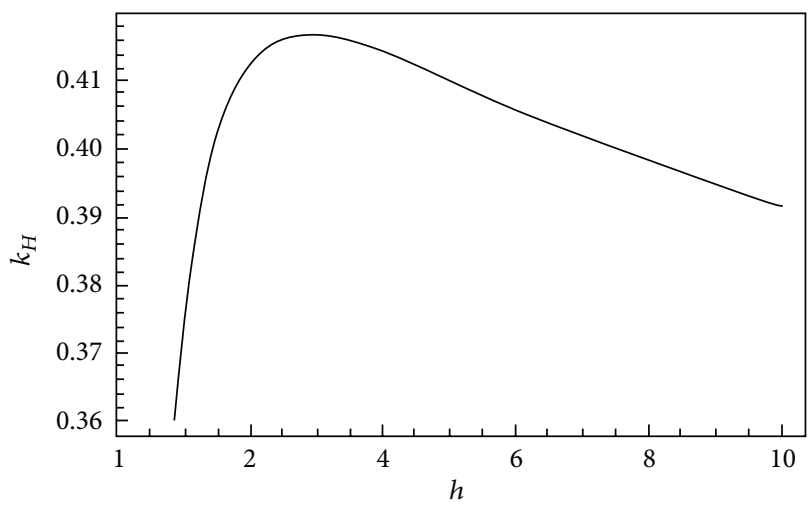

Figure 3: Theoretical dependence of the Huggins coefficient on the draining parameter $h$, calculated from (10).

results for the intrinsic viscosity presented in Table 1 are excellently described by this equation with the constants $\alpha=$ $162.7 \mathrm{~K} \mathrm{~mL} / \mathrm{mg}$ and $\beta=-0.443 \mathrm{~mL} / \mathrm{mg}$.

\section{Conclusion}

In conclusion, we have studied, both experimentally and theoretically, the high molecular weight Poly(2-ethyl-2oxazoline) aqueous solutions. The viscometric measurements allowed us to determine the intrinsic viscosity and other important parameters of the solutions around the temperature $25^{\circ} \mathrm{C}$, at which, according to the literature, the solutions are at theta condition. The experimental results were interpreted within a model that joins the Rouse and Zimm models of the polymer dynamics and thus goes back to the classical Kirkwood and Riseman theory [27]. Contrary to the Flory theory of steady-state transport processes in dilute polymer solutions [1], the ratio of the intrinsic viscosity $[\eta]$ to $R_{G}^{3}$, where $R_{G}$ is the gyration radius, is not a universal constant for long polymer chains. The polymer characteristics, such as the diffusion coefficient of the coils or their relaxation times of internal modes, depend on the draining parameter connected with the permeability of the coils with respect to the solvent and the strength of the hydrodynamic interactions between the polymer segments. The influence of draining to the viscosity and dimensions of the chain macromolecules in different solvents has been considered in a number of works [45-48]. For the study of polymers in good solvents see the recent work [49] where it was shown that the draining effects are responsible for the decrease of the viscometric unperturbed dimensions parameter of a polymer in the blob theta conditions, if compared with such a parameter for a whole polymer in the theta solvent. The question how much the polymer chain is sensitive to the draining is still puzzling $[49,50]$. Although several experiments indicated nonuniversality in the polymer behavior, the draining effects are usually ignored by the experimentalists and the polymers are considered in the nondraining limit, which in the presented model corresponds to the infinitely large draining parameter $h$. We have calculated the polymer parameters as functions of $h$ and the solution concentration $c$. The 
obtained spectrum of the polymer internal modes allowed us to calculate the solution viscosity and, using it, the intrinsic viscosity. The calculated Huggins coefficient was compared to its experimentally determined value. We have found that for $h$ corresponding to the maximum of the peak in the theoretically predicted function $k_{H}(h)$ the experimental value of $k_{H}$ exactly agrees with the experiment. This happens at the temperature $T=20^{\circ} \mathrm{C}$. Since the theory is built for the theta condition, we propose that the theta temperature for PEOX is $20^{\circ} \mathrm{C}$ rather than $25^{\circ} \mathrm{C}$ known from the previous studies. In our opinion, further investigations are needed to solve this controversy. This can be done, for example, by studying the dependence of the intrinsic viscosity on the polymer mass at different temperatures. We have also estimated the gyration and hydrodynamic radii of the polymer coils. Finally, we have shown that our experimental results very well correspond to the assumption that the shear viscosity of the solutions is a thermally activated process described by the Arrhenius equation.

\section{Conflict of Interests}

The authors declare that there is no conflict of interests regarding the publication of this paper.

\section{Acknowledgments}

This work was supported by the Slovak Ministry of Education Agency for the Structural Funds of the EU within the projects 26220120097 and 26220220182 and by the Grant VEGA $1 / 0348 / 15$.

\section{References}

[1] P. J. Flory, Principle of Polymer Chemistry, Cornell University Press, Ithaca, NY, USA, 1953.

[2] M. Doi and S. F. Edwards, The Theory of Polymer Dynamics, Oxford University Press, New York, NY, USA, 1986.

[3] H. Yang, Y. Yan, P. Zhu, H. Li, Q. Zhu, and C. Fan, "Studies on the viscosity behavior of polymer solutions at low concentrations," European Polymer Journal, vol. 41, no. 2, pp. 329-340, 2005.

[4] J. Tóthová and V. Lisý, "Relaxation times of flexible polymer chains in solution from non-conventional viscosity measurements," The Open Macromolecules Journal, vol. 4, pp. 26-31, 2010.

[5] R. G. Larson, "The rheology of dilute solutions of flexible polymers: progress and problems," Journal of Rheology, vol. 49, no. 1, pp. 1-70, 2005.

[6] H. Yamakawa, Modern Theory of Polymer Solutions, Harper's Chemistry Series, Harper and Row, New York, NY, USA, 2001.

[7] K. Kamide and S. Matsuda, "A method for determining the Flory theta temperature and entropy parameter of singleor multicomponent-polymer-single solvent systems from the critical solution point data," Polymer Journal, vol. 16, no. 11, pp. 825-837, 1984.

[8] K. Bechekh and N. Ghaouar, "Rheological properties of polyethylene glycol (PEG 35000): an interpretation of a negative intrinsic viscosity and a high huggins coefficient value," Journal of Macromolecular Science Part B: Physics, vol. 53, no. 3, pp. 391397, 2014.
[9] W. B. Russel, D. A. Saville, and W. R. Schowalter, Colloidal Dispersions, Cambridge University Press, Cambridge, UK, 1989.

[10] J. Tothova and V. Lisy, "Intrinsic viscosity of PVP polymers in extremely diluted solutions," E-Polymers, vol. 13, no. 1, pp. 243248, 2013.

[11] J. Tóthová, M. Timko, P. Kopčanský, and V. Lisý, "Search for anomalous temperature behavior of the viscosity of polyethylene glycol solutions," International Journal of Thermophysics, vol. 35, no. 11, pp. 2150-2157, 2014.

[12] T. T. Chiu, B. P. Thill, and W. J. Fairchok, "Poly (2-ethyl-2oxazoline): a new water-and organic soluble adhesive," in WaterSoluble Polymers, vol. 213 of Advances in Chemistry, chapter 23, pp. 425-433, American Chemical Society, Washington, DC, USA, 1986.

[13] V. Aseyev, H. Tenhu, and F. M. Winnik, "Non-ionic thermoresponsive polymers in water," Advances in Polymer Science, vol. 242, no. 1, pp. 29-89, 2011.

[14] A. M. Bernard, Molecular modeling of poly(2-ethyl-2oxazoline) [Ph.D. thesis], Georgia Institute of Technology, Atlanta, Ga, USA, 2008.

[15] H. Schlaad and R. Hoogenboom, "Poly(2-oxazoline)s and related pseudo-polypeptides," Macromolecular Rapid Communications, vol. 33, no. 19, p. 1599, 2012.

[16] Y. Han, Z. He, A. Schulz et al., "Synergistic combinations of multiple chemotherapeutic agents in high capacity poly(2oxazoline) micelles," Molecular Pharmaceutics, vol. 9, no. 8, pp. 2302-2313, 2012

[17] N. Adams and U. S. Schubert, "Poly(2-oxazolines) in biological and biomedical application contexts," Advanced Drug Delivery Reviews, vol. 59, no. 15, pp. 1504-1520, 2007.

[18] J. C. M. E. Bender, R. Hoogenboom, and P. A. A. van Vliet, "Drug delivery system comprising Polyoxazoline and a bioactive agent," U.S. Patent No. US 8,642,080 B2, 2014.

[19] V. G. Kadajji and G. V. Betageri, "Water soluble polymers for pharmaceutical applications," Polymers, vol. 3, no. 4, pp. 19722009, 2011.

[20] A. Baumgaertel, C. Weber, K. Knop, A. Crecelius, and U. S. Schubert, "Characterization of different poly(2-ethyl2-oxazoline)s via matrix-assisted laser desorption/ionization time-of-flight tandem mass spectrometry," Rapid Communications in Mass Spectrometry, vol. 23, no. 6, pp. 756-762, 2009.

[21] F. P. Chen, A. E. Ames, and L. D. Taylor, "Aqueous solutions of poly(ethyloxazoline) and its lower consolute phase transition," Macromolecules, vol. 23, no. 21, pp. 4688-4695, 1990.

[22] C. H. Chen, J. E. Wilson, R. M. Davis, W. Chen, and J. S. Riffle, "Measurement of the segmental adsorption energy of poly(2-ethyl-2-oxazoline) on silica in water and ethanol," Macromolecules, vol. 27, no. 22, pp. 6376-6382, 1994.

[23] B. H. Zimm, "Dynamics of polymer molecules in dilute solution: viscoelasticity, flow birefringence and dielectric loss," The Journal of Chemical Physics, vol. 24, no. 2, pp. 269-278, 1956.

[24] P. E. Rouse Jr., "A theory of the linear viscoelastic properties of dilute solutions of coiling polymers," The Journal of Chemical Physics, vol. 21, no. 7, pp. 1272-1280, 1953.

[25] V. Lisy, J. Tothova, and A. V. Zatovsky, "Long-time dynamics of Rouse-Zimm polymers in dilute solutions with hydrodynamic memory," Journal of Chemical Physics, vol. 121, no. 21, pp. 1069910706, 2004.

[26] V. Lisy, J. Tothova, and A. V. Zatovsky, "The joint RouseZimm theory of the dynamics of polymers in dilute solutions," Condensed Matter Physics, vol. 9, no. 1, pp. 95-102, 2006. 
[27] J. G. Kirkwood and J. Riseman, "The intrinsic viscosities and diffusion constants of flexible macromolecules in solution," The Journal of Chemical Physics, vol. 16, no. 6, pp. 565-573, 1948.

[28] A. Dondos, C. Tsitsilianis, and G. Staikos, "Viscometric study of aggregation phenomena in polymer dilute solutions and determination of the critical concentration $c^{* *}$, Polymer, vol. 30, no. 9, pp. 1690-1694, 1989.

[29] A. Dondos and D. Papanagopoulos, "Difference between the dynamic and static behaviour of polymers in dilute solutions: 1 . The critical concentration $c^{* *}$, Polymer, vol. 36, no. 2, pp. 365368, 1995.

[30] E. Cherif, O. Zoghlami, and T. Othman, "Investigation of critical concentrations of poly(vinyl pyrrolidone) in $\mathrm{N}, \mathrm{N}$ dimethylformamide by a viscosity technique," Physics and Chemistry of Liquids, vol. 53, no. 1, pp. 75-83, 2015.

[31] T. Konishi, T. Yoshizaki, and H. Yamakawa, "On the 'universal constants' $\rho$ and $\Phi$ of flexible polymers," Macromolecules, vol. 24, no. 20, pp. 5614-5622, 1991.

[32] N. Sawatari, T. Yoshizaki, and H. Yamakawa, "Dynamic structure factor of polystyrene and poly(methyl methacrylate) in $\vartheta$ solvents," Macromolecules, vol. 31, no. 13, pp. 4218-4222, 1998.

[33] J. Tothova, B. Brutovsky, and V. Lisy, "Draining and concentration effects in dynamic light scattering from nonentangled polymers in solution," Laser Physics, vol. 17, no. 4, pp. 443-447, 2007.

[34] H. C. Brinkman, "A calculation of the viscous force exerted by a flowing fluid on a dense swarm of particles," Applied Scientific Research A, vol. 1, no. 1, pp. 27-34, 1949.

[35] P. Debye and A. M. Bueche, "Intrinsic viscosity, diffusion, and sedimentation rate of polymers in solution," The Journal of Chemical Physics, vol. 16, no. 6, pp. 573-579, 1948.

[36] J. Tothova, B. Brutovsky, and V. Lisy, "Single monomer dynamics in DNA polymers," Czechoslovak Journal of Physics, vol. 55, no. 2, pp. 221-227, 2005.

[37] J. Tothova, B. Brutovsky, and V. Lisy, "Monomer dynamics in single- and double-stranded DNA coils," European Physical Journal E, vol. 24, no. 1, pp. 61-67, 2007.

[38] A. Y. Grosberg and A. R. Khokhlov, Statistical Physics of Macromolecules, Polymers and Complex Materials, American Institute of Physics Press, Woodbury, NY, USA, 1994.

[39] M. Abramowitz and I. A. Stegun, Handbook of Mathematical Functions, National Bureau of Standards, Washington, DC, USA, 1972.

[40] M. Muthukumar, "The series expansion for the concentration dependence of relaxation times for dilute polymer solutions," The Journal of Chemical Physics, vol. 79, no. 8, pp. 4048-4055, 1983.

[41] Wolfram Research, Mathematica, Version 10.0.1.0, Wolfram Research, Champaign, Ill, USA, 2014.

[42] A. Einstein, "Eine neue Bestimmung der Moleküldimensionen," Annalen der Physik, vol. 19, supplement, pp. 289-306, 1906.

[43] X. Ye, J. Yang, and J. Ambreen, "Scaling laws between the hydrodynamic parameters and molecular weight of linear poly(2-ethyl-2-oxazoline)," RSC Advances, vol. 3, no. 35, pp. 15108-15113, 2013.

[44] W. R. Moore, "Viscosity-temperature relationships for dilute solutions of high polymers," Nature, vol. 191, no. 4795, pp. 12921293, 1961.

[45] G. C. Berry, "Thermodynamic and conformational properties of polystyrene. II. Intrinsic viscosity studies on dilute solutions of linear polystyrenes," The Journal of Chemical Physics, vol. 46, no. 4, pp. 1338-1352, 1967.
[46] A. Dondos, "A new relation between the intrinsic viscosity and the molecular mass of polymers derived from the blob model: determination of the statistical segment length of flexible polymers," Polymer, vol. 42, no. 3, pp. 897-901, 2001.

[47] A. Dondos, "Denaturated proteins: draining effect and molecular dimensions," Physica B, vol. 405, no. 17, pp. 3572-3575, 2010.

[48] A. Dondos, "Treating any polymer dissolved in water as polymer presenting a draining effect," Polymer Bulletin, vol. 67, no. 2, pp. 333-342, 2011.

[49] A. Dondos, "Unperturbed dimensions of polymers revisited through the blob theory: the viscometric expansion factor," $E$ Polymers, vol. 13, no. 1, pp. 144-152, 2014.

[50] J. J. H. Mulderije and H. L. Jalink, "Least-draining limit of linear flexible chain molecules," Macromolecules, vol. 20, no. 5, pp. 1152-1158, 1987. 

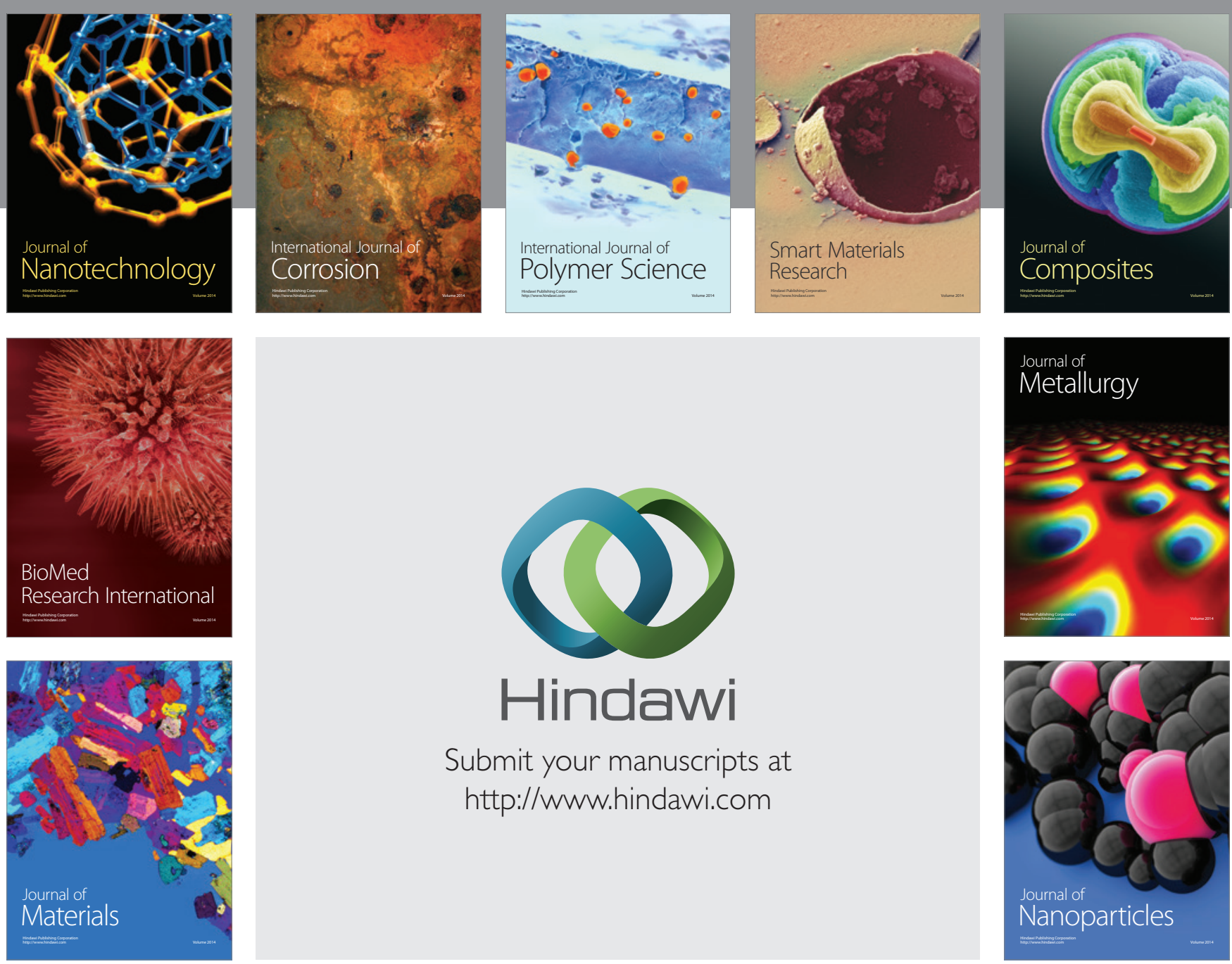

Submit your manuscripts at http://www.hindawi.com
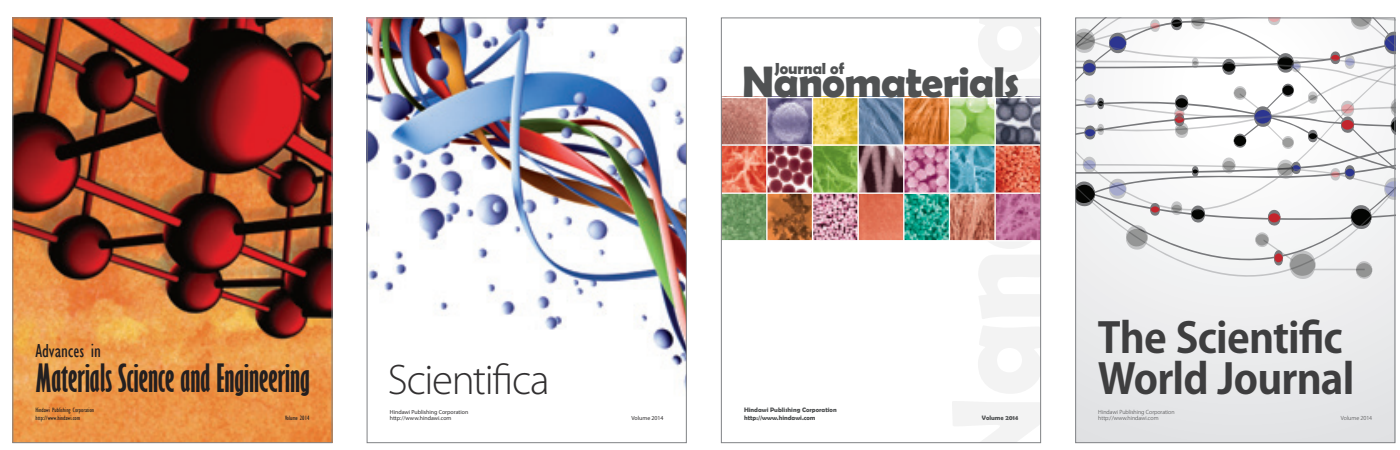

\section{The Scientific World Journal}
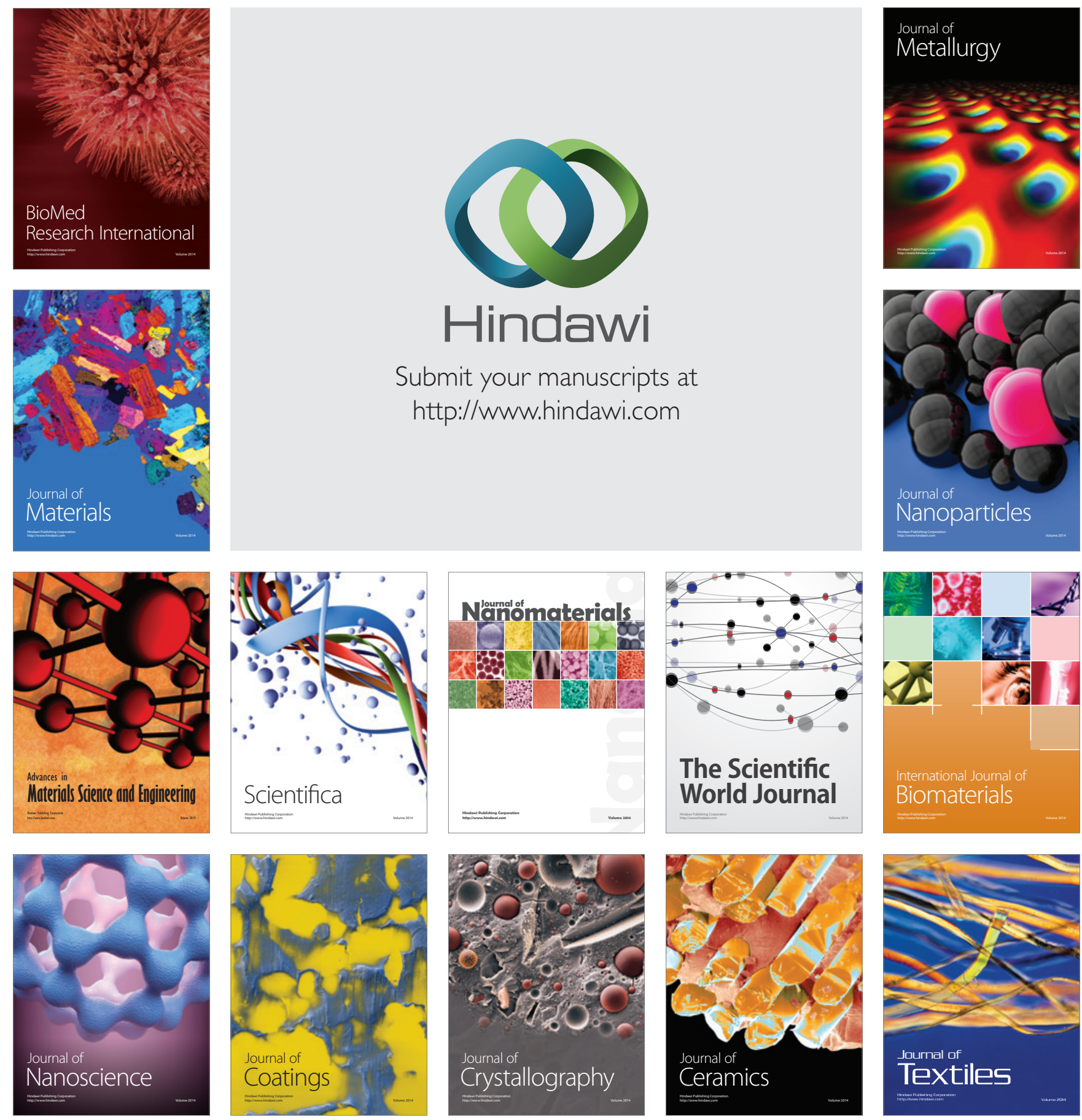